\title{
Type 1 Diabetes and the HLA-D Locus
}

\author{
J. A. Sachs, A. G. Cudworth, D. Jaraquemada, A. N. Gorsuch, and H. Festenstein \\ Departments of Diabetes and Immunology, St. Bartholomew's Hospital and the London Hospital Medical Colleges, London, England
}

Summary. HLA-D specificities have been investigated in 58 classical Type 1 diabetics and 43 healthy subjects. Both groups were selected according to the HLA-B locus antigens which are known to have a significant positive or negative association with the disease. The results indicate that (1) the primary association of the disease is with HLA-DW3, (2) the increased frequency of DW4 in diabetics with rare exception is co-existent with the presence of DW3, (3) the low frequency of DW2 is secondary to the increase in DW3 and/or DW4, and is not consistent with a primary 'protective' role. It is suggested that these data support the hypothesis of interaction between HLA-linked genes operating by separate mechanisms to confer the susceptibility to young onset Type 1 diabetes (Type 1A).

Key words: Genetic susceptibility in diabetes, Type 1 diabetes, the HLA system in diabetes, HLA-DW typing.

There is good evidence that the major genetic susceptibility to insulin dependent (Type 1) diabetes is encoded by genes within the HLA complex and is associated with an increased incidence of particular HLA specificities [1-6]. Thus, there is a two to three fold increased relative risk of developing the disease in people with the antigens HLA-B8 or HLA-B15. Preliminary evidence for a higher relative risk in subjects with HLA-DW3 and HLA-DW4 has been reported $[5,6]$. From the established linkage disequilibrium between HLA-A1-B8-DW3 and between HLA-A2-B15-CW3-DW4 in Caucasoids, it has been possible to construct two corresponding axes of susceptibility in Type 1 diabetes $[2,4,5]$.
Furthermore, subjects who are positive for determinants from both axes (e.g. HLA-B8, B15), have an additive risk [1-6].

One interpretation of these findings is that there are two HLA-linked genes operating by different mechanisms which confer the susceptibility [4-7]. In contrast, Rubinstein et al. [8] have argued that the predisposition is more compatible with recessive inheritance because they found that diabetic siblings were identical for the same HLA-D locus determinants.

It has also been shown that HLA-B7 and HLADW2 have a very low frequency in Type 1 diabetics, which has led to the idea that there may be important genes in linkage disequilibrium with the HLA-DW2B7 axis which confer "protection" or "resistance" to the development of the disease $[4,6]$.

To test these hypotheses further, we have studied Type 1 diabetic patients and controls who were positive for the relevant HLA-B locus antigens, i.e. HLA-B7, HLA-B8 and HLA-B15.

\section{Patients and Methods}

Fifty eight classical Type 1 diabetics (age of onset 2-16 years; mean 8.8 years) and 43 healthy subjects were selected and tested simultaneously. The diabetics were chosen from a current prospective familiy study.

Seven HLA-D locus specificities (DW1-DW7) were determined by the mixed lymphocyte culture (MLC) technique according to the standards proposed by the 1977 Histocompatibility Workshop [9]. The homozygous typing cells used for the definition of DW2 and DW3 were accredited by the appropriate reference laboratories (information available from J. A.S.). The definition of DW4 was assessed by two new homozygous typing cells, both of which gave high correlation coefficients with cells used in the 1977 Histocompatibility Workshop [9] to define the same specificity. Definition of DW2 was based on a typing response with the homozygous typing cells according to the scoring method of Mendel et al. [10]. Since this method is suitable for responder panels 
Table 1. Distribution of HLA-DW specificities in relation to the HLA-B locus antigens in diabetics and controls

\begin{tabular}{|c|c|c|c|c|c|c|}
\hline \multirow[t]{2}{*}{ HLA-D locus determinants } & \multicolumn{6}{|c|}{ HLA-B locus determinants } \\
\hline & $\begin{array}{l}\overline{\mathrm{B} 8, \mathrm{X}^{\mathrm{b}}} \\
\text { Diabetics }\end{array}$ & Controls & $\begin{array}{l}\mathrm{B} 15, \mathrm{X}^{\mathrm{a}} \\
\text { Diabetics }\end{array}$ & Controls & $\begin{array}{l}\mathrm{B} 7, \mathrm{X}^{\mathrm{b}} \\
\text { Diabetics }\end{array}$ & Controls \\
\hline DW2, X & - & - & - & - & 4 & 4 \\
\hline DW3, X & 12 & 10 & - & - & 6 & 2 \\
\hline DW4, X & - & 1 & 1 & 7 & 1 & 1 \\
\hline DW3, DW4 & 12 & 2 & 18 & 1 & 9 & - \\
\hline $\left.\begin{array}{l}\text { DW2, DW3 } \\
\text { DW2, DW4 }\end{array}\right\}$ & - & 3 & - & - & 2 & 5 \\
\hline Others & - & 5 & - & 4 & 4 & 4 \\
\hline Total & 24 & 21 & 19 & 12 & 26 & 16 \\
\hline
\end{tabular}

a Includes subjects who are HLA-B8, B15: Diabetics $(n=6)$; Controls $(n=1)$

${ }^{b}$ Includes subjects who are HLA-B8, B7: Diabetics $(n=5)$; Controls $(n=5)$

$\mathrm{X}=$ another specificity other than HLA-B8, HLA-B15 or HLA-B7 respectively

Table 2. Analysis of HLA-DW specificities in diabetics and controls

\begin{tabular}{lcccrc}
\hline HLA-D & $\begin{array}{l}\text { Diabetics } \\
(\mathrm{n}=58)\end{array}$ & $\begin{array}{l}\text { Controls } \\
(\mathrm{n}=43)\end{array}$ & $\begin{array}{l}\text { Chi-squared } \\
\text { (Yates) }\end{array}$ & P value $^{\mathrm{b}}$ & $\begin{array}{c}\mathrm{R}_{\text {Relative Risk }}^{\mathrm{a}} \\
\text { (Haldane) }\end{array}$ \\
\hline DW3, X & $47(81 \%)$ & $14(33 \%)$ & 22.3 & $6.2 \times 10^{-6}$ & 8.4 \\
DW4, X & $32(55 \%)$ & $15(35 \%)$ & 3.3 & 0.21 & 2.3 \\
DW3, DW4 & $29(50 \%)$ & $2(5 \%)$ & 21.8 & $2.1 \times 10^{-6}$ & 16.6 \\
DW2, X & $6(10 \%)$ & $10(23 \%)$ & 2.2 & 0.49 & 0.4 \\
\hline
\end{tabular}

a the relative risk applies only to the selected data as described in 'patients and methods'

$b$ the $p$ values shown are corrected for the number of specificities tested for

$\mathrm{X}=$ any other specificity

with relatively few typing responses, the conventional double normalised values method [11] was modified for the identification of the DW3 and DW4 specificities where they appeared to occur with a frequency of more than $50 \%$ of the test responder panels. Thus, an individual with a value of less than $30 \%$ of the median of three negative control cells was considered to be DW3 or DW4 positive.

The relative risks were computed by the Woolf method [12] (modified by Haldane). The $\mathrm{p}$ values were obtained by the FisherIrwin method and corrected for the number of specificites tested for.

\section{Results}

The frequencies of HLA-DW2, HLA-DW3 and HLA-DW4 in relation to the HLA-B locus antigens are shown in Table 1 . Thus, all the HLA-B8 positive diabetics were DW3 compared with $57 \%$ of the controls. All HLA-B15 positive diabetics were HLADW4 and 18 out of 19 (95\%) had HLA-DW3 as well, though only six of these were HLA-B8. In the control group, 8 out of 12 had HLA-DW4, but in contrast only one subject ( $8 \%$ ) had both HLA-DW3 with HLA-DW4.

Of 26 HLA-B7 positive diabetics, only $6(23 \%)$ were HLA-DW2 compared with nine out of 16 $(56 \%)$ in the controls. Two of the HLA-DW2 positive diabetics also had HLA-DW3, but it is of par- ticular interest that 18 out of $26(69 \%)$ of this group possessed HLA-DW3 and/or HLA-DW4.

In Table 2 we compared the overall frequencies of HLA-DW2, HLA-DW3 and HLA-DW4 between the diabetics and the controls. Thus, in this selected series, there is a highly significant increased frequency of HLA-DW3, $X\left(p=6.2 \times 10^{-6}\right.$; relative risk $=8.4$ ), whereas the frequency of HLA-DW4, $X$ is not significant in the diabetics when corrected for the number of specificities tested for $(\mathrm{p}=0.21$; relative risk $=2.3$ ). However, the most interesting feature is the striking co-existence of HLA-DW3 with HLA-DW4 in the diabetics $\left(\mathrm{p}=2.1 \times 10^{-6}\right.$; relative risk $=16.6$ ). Although there is a low relative risk in respect of HLA-DW2 (0.4), this was not significantly different from the controls $(p=0.49)$.

\section{Discussion}

This study was performed on individuals selected for the presence of particular HLA-B locus antigens in order to investigate the genetic basis of the HLA-D locus association with Type 1 diabetes. The data strongly support the concept that the HLA-DW3 determinant is the primary association with the dis- 
ease [4-6]. There is also a significant increase in the frequency of HLA-DW4. Thus, the associations first observed with HLA-B8 and HLA-B15 are undoubtedly secondary to the established linkage disequilibrium with HLA-DW3 and HLA-DW4 respectively. A striking feature of the present study is the co-existence of HLA-DW4 with HLA-DW3 in all the patient groups studied including those selected for the presence of HLA-B7 (Table 1). Only two diabetic patients possessed HLA-DW4 alone. Therefore, we would claim that the major genetic susceptibility to Type 1 diabetes is conferred by a gene or genes in linkage disequilibrium with HLA-DW3.

An important question is whether these data support the theory of autosomal recessive inheritance or are more consistent with the concept of HLA gene interaction. In respect to the latter, it might be proposed that a gene in linkage disequilibrium with HLA-DW4 is on its own a weak susceptibility factor, but when present with the major susceptibility gene (in linkage disequilibrium with HLA-DW3), the result is a markedly enhanced risk of beta cell destruction. We feel our results support this hypothesis.

If we consider the hypothesis of an autosomal recessive inheritance, it would be expected that there should be an increased frequency of subjects with HLA-DW3 alone, or HLA-DW4 alone, suggesting possible homozygosity for the same HLA-D linked 'diabetogenic' gene. In this series there were no possible homozygous HLA-DW4 diabetics, and overall there were only nine possible HLA-DW3 homozygotes $(15.5 \%)$. This is the same prevalence of possible HLA-DW3 and HLA-DW4 homozygotes observed in the healthy subjects $(16 \%)$. Future studies of the HLA-D and the closely related serologically defined HLA-DR specificities in HLA genotyped families may help to clarify this point.

Although the frequency of HLA-DW2 in the HLA-B7 diabetic group was low (10\% v. $23 \%$ in controls, $p=0.49$ ), our data indicate that this is secondary to the increased frequency of HLA-DW3. This is against the hypothesis that there are genes in linkage disequilibrium with the HLA-DW2-B7 axis which confer a primary 'protection' or 'resistance'.

If the theory of gene interaction is correct, we can speculate that different HLA-linked genes operating by separate mechanisms contribute to the pathogenesis of beta cell destruction in Type 1 diabetes. One of these genes (in linkage disequilibrium with DW3) may control the immune response to environmental agents (e.g. viruses) whose propensity for initiating damage to the beta cell membrane is determined by other genes (in linkage disequilibrium with DW4). All the diabetics in this study were of young onset in whom there is male preponderance, rapid development of beta cell decompensation and transient appearance of islet cell antibodies [5].

Acknowledgements. We thank Bridget Watson for the invaluable assistance and also Dr. Eva Wolf and Janet Pritchard for the HLAA, B, C studies.

This work was supported by the Medical Research Council, the British Diabetic Association, the Juvenile Diabetes Foundation of America, and the Joint Research Board of St. Bartholomew's Hospital. D. J. is supported by the Arthritis and Rheumatism Research Council.

\section{References}

1. Nerup, J., Cathelineau, C., Seignalet, J., Thomsen, M.: In: HLA and disease. Dausset, J., Svejgaard, A. (Eds.), pp. 149-167. Cophenhagen: Munksgaard 1976

2. Cudworth, A. G.: Type 1 diabetes mellitus. Diabetologia 14, 281-291 (1978)

3. Thomsen, M., Platz, P., Andersen, O. O., Christy, M., Lyngsøe, J., Nerup, J., Rasmussen, K., Ryder, L. P., Nielsen, L. S., Svejgaard, A.: MLC typing in juvenile diabetes mellitus and idiopathic Addison's disease. Transplant. Rev. 22, 120-125 (1975)

4. Cudworth, A. G., Festenstein, H.: HLA genetic heterogeneity in diabetes mellitus. Br. Med. Bull. 34, 285-289 (1978)

5. Cudworth, A. G., Bottazzo, G. F., Doniach, D.: Genetic and immunological factors in Type 1 diabetes mellitus. In: Immunology of diabetes. Irvine, W.J. (Ed.). Edinburgh: Teviot Publications 1979

6. Nerup, J., Christy, M., Kromann, H., Andersen, O. O., Platz, P., Ryder, L. P., Thomsen, M., Svejgaard, A.: Genetic susceptibility and resistance to insulin dependent diabetes mellitus (IDDM). In: Immunology of diabetes. Irvine, W. J. (Ed.). Edinburgh: Teviot Publications 1979

7. Festenstein, H., Demant, P.: In: HLA and H-2. Festenstein, H., Demant, P. (Eds.), p. 169. London: Edward Arnold 1978

8. Rubinstein, P., Suciu-Foca, N., Nicholson, J. F.: Genetics of juvenile diabetes mellitus. A recessive gene closely linked to HLA D with 50 per cent penetrance. N. Engl. J. Med. 297, 1036-1040 (1977)

9. Bodmer, W. F., Batchelor, J. R., Bodmer, J. B., Festenstein, H. (Eds.): Histocompatibility testing 1977, pp. 85-145. Copenhagen: Munksgaard 1977

10. Mendel, N. R., Guppy, D., Bodmer, W. F., Festenstein, H.: In: Histocompatibility testing 1977. Bodmer, W. F., Batchelor, J. R., Bodmer, J. B., Festenstein, H. (Eds.), p. 90. Copenhagen: Munksgaard 1977

11. Ryder, L. P., Thomsen, M., Platz, P., Svejgaard, A.: In: Histocompatibility testing 1975 . Kissmeyer-Nielsen, F. (Ed.), p. 557. Copenhagen: Munksgaard 1975

12. Woolf, B.: On estimating the relation between blood groups and disease. Ann. Hum. Genet. 19, 251-253 (1955)

Received: May 21, 1979,

and in revised form: July 31, 1979

Dr. A. G. Cudworth

Medical Unit

St. Bartholomew's Hospital

West Smithfield

London EC1A 7BE

England 